\title{
The possibility of simplified modelling of radiation heat transfer within a steel porous charge
}

\author{
Rafał Wyczółkowski ${ }^{1, *}$, Tomasz Wyleciał ${ }^{1}$, and Marek Gała ${ }^{2}$ \\ ${ }^{1}$ Czestochowa University of Technology, Faculty of Production Engineering and Materials Technology, Department of Industrial \\ Furnaces and Environmental Protection, Al. Armii Krajowej 19, 42-200 Czestochowa, Poland \\ ${ }^{2}$ Czestochowa University of Technology, Faculty of Electrical Engineering, Institute of Electric Power Engineering, \\ Al. Armii Krajowej 17, 42-200 Czestochowa, Poland
}

\begin{abstract}
The article refers to the problem of calculating the effective thermal conductivity $k_{\text {ef }}$ of a steel porous charge. In proposed approach for each heat transfer mechanism, which occurs in the considered medium, the corresponding thermal resistance is assigned. The model values of the $k_{\text {ef }}$ coefficient were determined twice for the same input data (geometric dimensions, emissivity, temperature, thermal conductivity of steel and gas). The difference between successive calculations depended on the use of two different methods to determine the thermal radiation resistance $R_{\mathrm{rd}}$. In the first approach the resistance $R_{\mathrm{rd}}$ was calculated using the exact method and in the second approach the simplified, approximate method was used. The discrepancy between the obtained results of $k_{\mathrm{ef}}$ in both approaches provides the evidence to use the approximate method to determine the resistance $R_{\mathrm{rd}}$. A bundle of square steel sections were used to demonstrate the challenge. In the exact method, data regarding the temperature distribution within a single profile was used. These data were obtained based on experimental research using a guarded hot plate apparatus. The calculations were performed using a temperature range between $200^{\circ} \mathrm{C}$ and $800^{\circ} \mathrm{C}$ for two sections: $60 \times 60 \mathrm{~mm}$ and $80 \times 80 \mathrm{~mm}$ and three emissivities: $0.5,0.7$ and 0.9 .
\end{abstract}

\section{Introduction}

Heat treatment operations of steel products have significant bearing on all key performance metrics of the plant, i.e.: productivity, energy consumption, product quality and emission of pollutants. This causes that heat treatment parameters should be selected very carefully. In the metallurgical industry for control and design of heat treatment parameters special numerical models have been used for more than three decades [1-3]. Such models predict spatial and temporal changes in the temperature of the charge. One of the challenges of this prediction is having required knowledge about the thermal properties of the heated charge. This issue becomes particularly complicated when the charge with a porous structure (e.g bundles of long elements or wire coils) is treated. Such kind of charge is a granular medium with the gas phase filling the gaps in between the steel elements. Therefore, during the heat treatment the following complex processes take place simultaneously: conduction in steel, conduction in gas, contact conduction, free convection and thermal radiation. As a result, the effective thermal conductivity $k_{\text {ef }}$ becomes the key thermal property of the porous charge. This parameter is commonly used in the theory of porous media $[4,5]$.

The effective thermal conductivity of the porous material can be calculated by applying the model which is based on the analysis of thermal resistance for individual modes of heat transfer [6,7]. If the hightemperature process is applied to the above scenario, one of the heat transfer modes to be considered in the $k_{\mathrm{ef}}$ model is thermal radiation. The thermal resistance of radiation $R_{\mathrm{rd}}$ in this model can be calculated in two ways: exact and approximate. In the exact approach, resistance $R_{\mathrm{rd}}$ is determined using the radiosity method. The main challenge comes down to solving the system of equations. Obtaining a solution by this method requires knowledge on the temperature values of all the surfaces that close the space of radiative heat transfer. To obtain information on changes in the temperature distribution within the heated porous charge, experimental investigations are necessary. Therefore, this is a significant drawback of this method. This problem is eliminated in the approximate method as this utilises one simple equation in which only the average temperature is applied.

This paper compares the two methods of calculating the effective thermal conductivity of a porous charge. The first, thermal radiation resistance is determined using the exact method, the second the approximated method. The analysis was carried out for the porous charge of a steel square section bundle which is illustrated in Fig. 1. Due to the high porosity of this charge, the fraction of thermal radiation in the total heat transfer is particularly high in this case.

\footnotetext{
*Corresponding author: rwyczolkowski@wip.pcz.pl
} 


$$
q_{r d}=J_{1}-J_{2}
$$

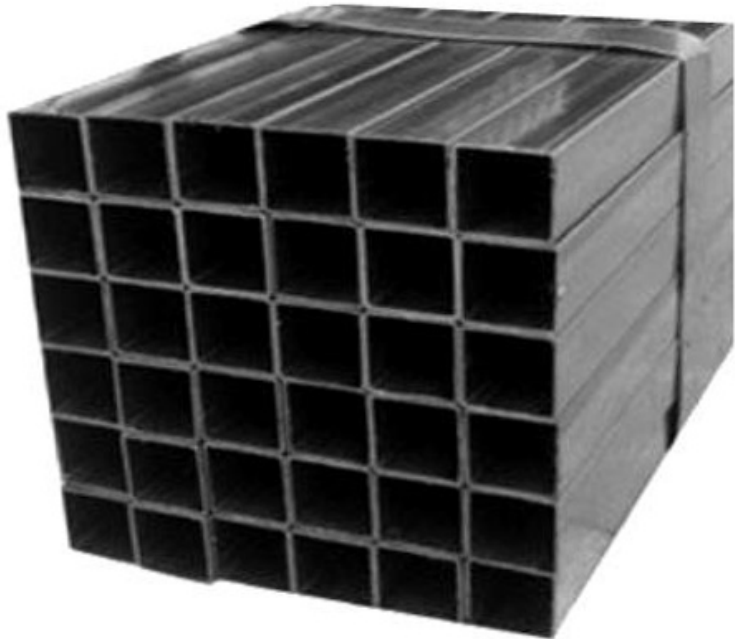

Fig. 1. Bundle of square sections as an example of a steel porous charge.

\section{Analysis and modelling}

When analyzing the thermal radiation in the area of the bundle, the process within each profile needs to be considered. This is radiation heat transfer in a threesurface enclosure as illustrated in Fig 2. It is assumed that the temperature of the bottom surface $A_{1}$ is $T_{1}$, the temperature of the top surface $A_{2}$ is $T_{2}$ and the temperature of the lateral surface $A_{3}$ is $T_{3}$ and $T_{1}>T_{3}>T_{2}$.

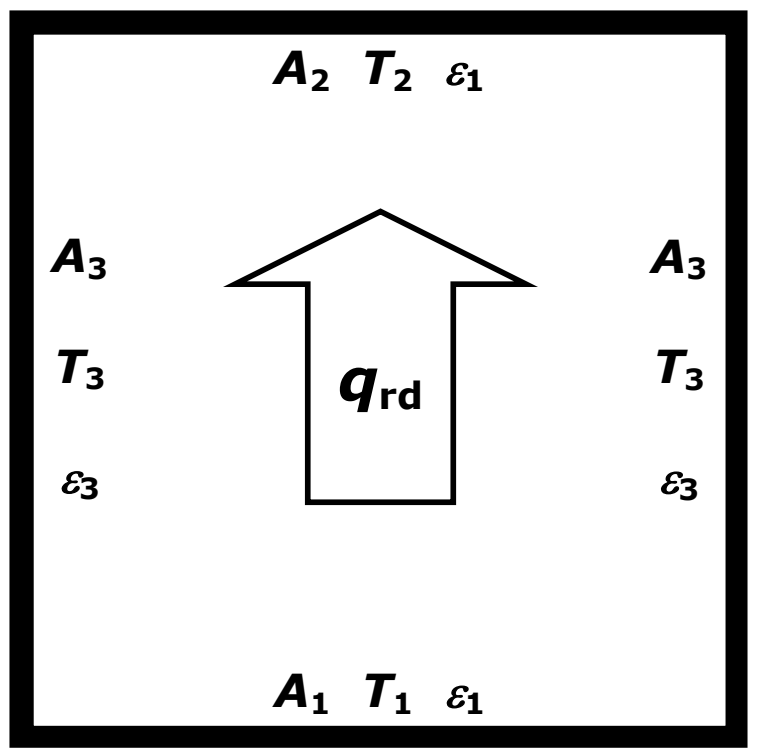

Fig. 2. The layout of the surfaces considered in the analysis of radiation heat transfer in the square section.

Since the enclosure is square the following relationship takes place: $A_{1}=A_{2}=0.5 A_{3}$. It is also assumed that the surfaces of the enclosure are opaque, diffuse, grey and its radiative properties are the same and expressed by the emissivity $\varepsilon\left(\varepsilon_{1}=\varepsilon_{2}=\varepsilon_{3}\right)$. The net heat flux of radiation $q_{\mathrm{rd}}$ in this system is [8]: were $J_{1}$ and $J_{2}$ represents radiosity of the surfaces $A_{1}$ and $A_{2}$ respectively. The radiation resistance $R_{\mathrm{rd}}$ for this system is expressed by the following relationship:

$$
R_{r d}=\frac{T_{1}-T_{2}}{q_{r}}=\frac{T_{1}-T_{2}}{J_{1}-J_{2}}
$$

Therefore, the challenge in calculating the resistance $R_{\mathrm{rd}}$ in the exact method is to determine the radiosities $J_{\mathrm{i}}$ of all surfaces in the considered enclosure, which are described by the following equations:

$$
\begin{gathered}
J_{1}=E_{1}-F_{2-1} \rho J_{2}-F_{3-1} \rho J_{3} \\
J_{2}=E_{2}-F_{1-2} \rho J_{1}-F_{3-2} \rho J_{3} \\
J_{3}=E_{3}-F_{1-3} \rho J_{1}-F_{2-3} \rho J_{2}-F_{3-3} \rho J_{3}
\end{gathered}
$$

where $\rho=1-\varepsilon$ is surface reflectivity.

The emissive power per unit area of each surface $E_{\mathrm{i}}$ is described by the equation [8]:

$$
E_{i}=\varepsilon \sigma T_{i}^{4}
$$

where $\sigma$ is Stefan-Boltzmann constant.

The view factors $F_{\mathrm{i}-\mathrm{j}}$ used in equations (3) for the surface layout under consideration have the following values:

$$
\begin{gathered}
F_{1-2}=F_{2-1}=F_{3-3}=0.4 \\
F_{1-3}=F_{2-3}=0.6 \\
F_{3-1}=F_{3-2}=0.3
\end{gathered}
$$

In order to determine each radiosity, the system of equations (3) comes down to the matrix form:

$$
\mathbf{E}=\mathbf{C} \times \mathbf{J}
$$

where

$$
\begin{gathered}
\mathbf{E}=\left[\begin{array}{l}
E_{1} \\
E_{2} \\
E_{3}
\end{array}\right] \\
\mathbf{C}=\left[\begin{array}{ccc}
1 & -F_{2-1} \rho & -F_{3-1} \rho \\
-F_{1-2} \rho & 1 & -F_{3-2} \rho \\
-F_{1-3} \rho & -F_{2-3} \rho & 1-F_{3-3} \rho
\end{array}\right] \\
\mathbf{J}=\left[\begin{array}{l}
J_{1} \\
J_{2} \\
J_{3}
\end{array}\right]
\end{gathered}
$$

In the approximate approach, the resistance $R_{\mathrm{rd}}$ is calculated using a single equation. In this equation there is only one temperature $T_{\mathrm{m}}$ and its value is the average for the whole enclosure: 


$$
R_{r d}=\frac{X_{r d}}{4 \sigma T_{m}^{3}}
$$

where $X_{\text {rd }}$ is a dimensionless coefficient with the value depending on the emissivity and the shape, as well as the relative position of the surfaces that represent the boundaries for the space [9]. For square enclosure $X_{\mathrm{rd}}=1 / \varepsilon$.

The effective thermal conductivity of the analyzed charge is calculated using the definition of conduction resistance for the flat layer with dimension $l[10]$ :

$$
k_{e f}=\frac{l}{R_{t o}}
$$

where $R_{\mathrm{to}}$ is the total thermal resistance of the considered medium.

For the section bundle the $l$ represents the sum of section dimension in the direction of heat flow and the width of the gap between the individual sections. While resistance $R_{\mathrm{to}}$ is the sum of the section thermal resistance $R_{\mathrm{st}}$ and thermal resistance of the gap $R_{\mathrm{gp}}$ :

$$
R_{t o}=R_{s t}+R_{g p}
$$

The methodology for calculating the section thermal resistance $R_{\mathrm{st}}$ is described in the publication [11]. While calculating this parameter, the $R_{\mathrm{rd}}$ resistance needs to be taken into account. Whereas the gap thermal resistance $R_{\mathrm{gp}}$ is calculated by the polynomial [12]:

$$
R_{g p}=\left(B_{1} t^{2}+B_{2} t+B_{3}\right) \cdot 10^{-4}
$$

The values of coefficients $B_{\mathrm{i}}$ from this polynomial depend on the surface physical state of the adjacent sections.

\section{Results and discussion}

The calculations presented below were performed for two square sections: $60 \mathrm{~mm}$ and $80 \mathrm{~mm}$ with the wall thickness of $3 \mathrm{~mm}$ and three emissivities: $0.5,0.7$ and 0.9 . The temperatures of the individual section surfaces required for the calculation of the resistance $R_{\mathrm{rd}}$ in the exact method were obtained from experimental research. These measurements were taken while determining the effective thermal conductivity of the section bundles using the guarded hot plate apparatus [13]. During these experiments, temperatures $t_{1}$ and $t_{2}$ were captured using thin jacked thermocouples within the selected sections. Measurements points were located on opposite surfaces of the section, perpendicular to the direction of the heat flow. The results of the measurements were used to calculate the temperature differences $\Delta t=t_{1}-t_{2}$, which corresponds to the mean temperature $t_{\mathrm{m}}=0.5 \cdot\left(t_{1}+t_{2}\right)$. The values of the parameter $\Delta t$ in the function of the temperature $t_{\mathrm{m}}$ obtained for the sections 60 and $80 \mathrm{~mm}$ are shown in Fig. 3.

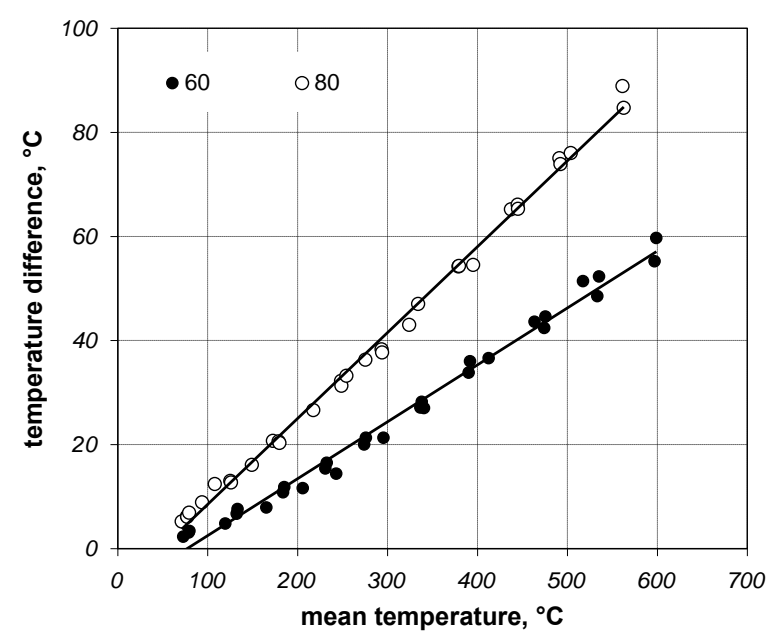

Fig. 3. Temperature differences within the 60 and $80 \mathrm{~mm}$ sections obtained from the experimental research.

The data presented in Fig. 3 was then approximated by linear regression equations. The following relationships were obtained for each section:

$$
\begin{aligned}
\Delta t_{60} & =0.1094 t_{m}-8.45 \\
\Delta t_{80} & =0.1681 t_{m}-8.94
\end{aligned}
$$

The equations (11) were used to determine the temperatures for each surfaces of the section $\left(T_{1}, T_{2}\right.$ and $T_{3}$ ) which are necessary for calculating the $R_{\mathrm{rd}}$ resistance in the exact method:

$$
\begin{gathered}
T_{1}=\left(t_{m}+273\right)+0.5 \Delta t \\
T_{2}=\left(t_{m}+273\right)-0.5 \Delta t \\
T_{3}=\left(t_{m}+273\right)
\end{gathered}
$$

Fig. 4 presents the results of radiation resistance calculations for the considered scenarios, which were obtained by both methods (exact and approximate). It was observed that the results for both sections using the same method (Figs. 4a and 4b) were very similar with only a very minor difference of $0.1 \%$ However, there are significant differences between the exact and the approximate methods for the same sections, with the exact method producing higher values. These differences in the results of both methods depend on the emissivity. When the emissivity decreases greater differences are observed.

In order to analyze the deviations in detail, the percentage excess of radiation resistance $\Delta R_{\mathrm{rd}}$ were calculated:

$$
\Delta R_{r d}=\frac{R_{r d-e x}-R_{r d-a p}}{R_{r d-a p}} \cdot 100 \%
$$

where $R_{\text {rdex }}$ is the resistance obtained using the exact method, whereas $R_{\text {rd-ap }}$ is the resistance obtained in the approximate method. 

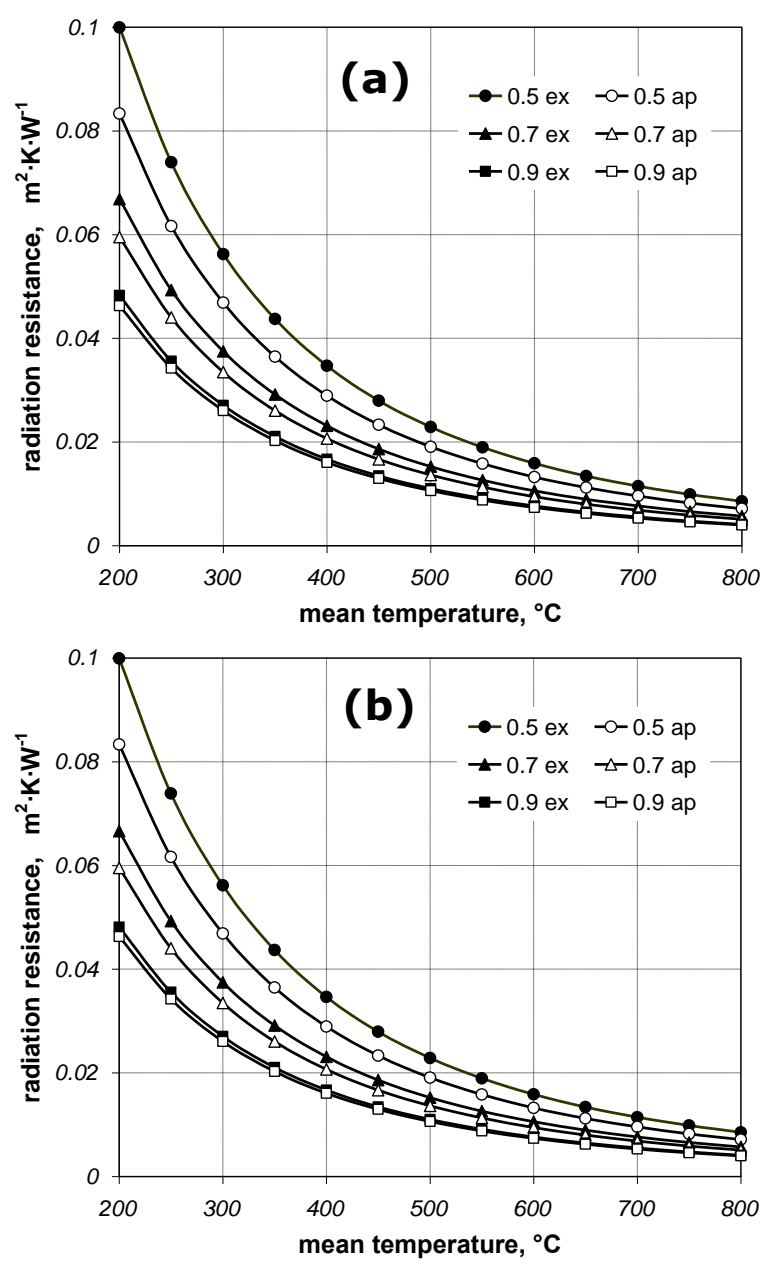

Fig. 4. Resistance $R_{\text {rd }}$ calculated by exact and approximate method for: a) section $60 \mathrm{~mm}$, b) section $80 \mathrm{~mm}$.

The results of the $\Delta R_{\mathrm{rd}}$ parameter obtained for the $80 \mathrm{~mm}$ section are presented in Fig. 5. It can be noted that this parameter is almost constant for each emissivity in a temperature function. Very similar results were obtained for the $60 \mathrm{~mm}$ section. This implies that for a given emissivity the average value of this parameter can be applied.

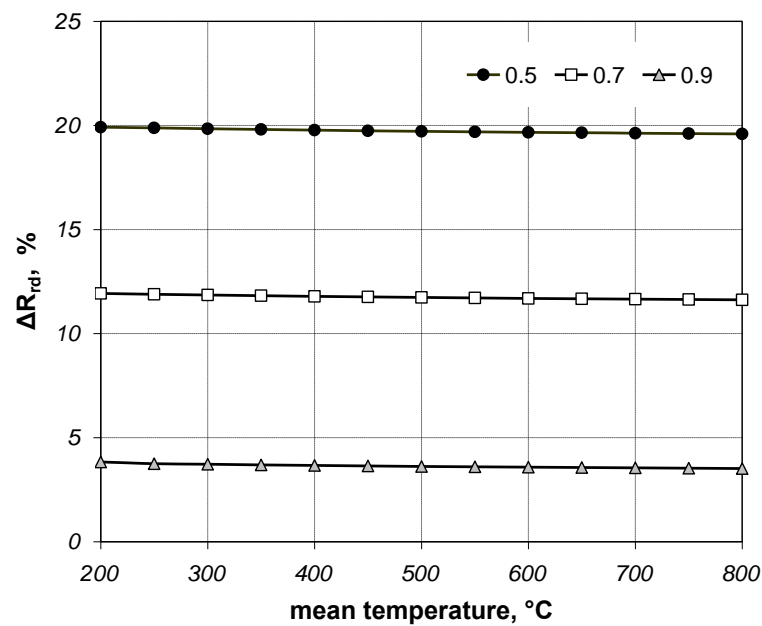

Fig. 5. Percentage excess of resistance $R_{\mathrm{rd}}$ for the $80 \mathrm{~mm}$ section.
Thus, the difference in $R_{\mathrm{rd}}$ resistance between the exact and the approximate method is reduced linearly with the increase in emissivity, reaching zero for the black body surface. However, for a square enclosure with an emissivity of 0.5 , the $R_{\text {rd-ex }}$ value is about $20 \%$ greater than the $R_{\text {rd-ap }}$ value.

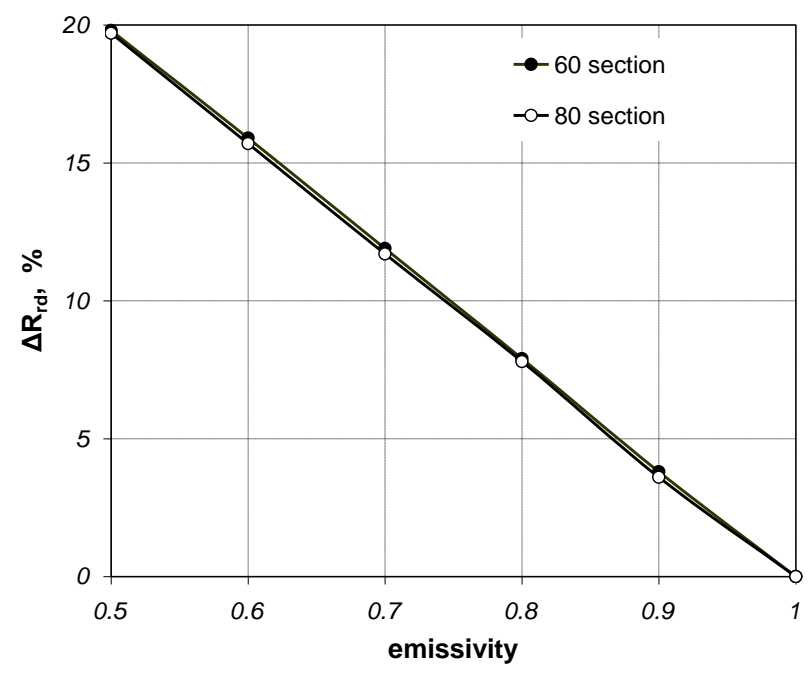

Fig. 6. The percentage excess of radiation resistance for the considered sections versus emissivity.

In line with the purpose of this article, it was decided to evaluate how the observed difference in $R_{\text {rd-ex }}$ and $R_{\text {rd-ap }}$ values affects the value of the effective thermal conductivity of the square section bundle. In these calculations it was assumed that the thermal conductivity of steel $k_{\mathrm{s}}$ and the thermal conductivity of gas phase $k_{\mathrm{g}}$ change with temperature in the following relationships:

$$
\begin{gathered}
k_{s}=1.2 \cdot 10^{-8} t^{3}-3.2 \cdot 10^{-5} t^{2}-1.2 \cdot 10^{-2} t+51.3 \\
k_{g}=-2.88 \cdot 10^{-8} t^{2}+8.05 \cdot 10^{-5} t+0.024
\end{gathered}
$$

Equation (14) describes changes in thermal conductivity of low-alloy steel with carbon content of $0.2 \%$, while equation (15) describes changes in thermal conductivity of air. These equations were determined through approximation of the literature data $[14,15]$.

The calculations of the coefficient $k_{\mathrm{ef}}$ were performed for the same scenarios as for the radiation resistance. To determine the gap thermal resistance $R_{\mathrm{gp}}$, in equation (10), the following values of $B_{\mathrm{i}}$ coefficients were used:

- $B_{1}=2.31 \cdot 10^{-5}$;

- $B_{2}=-0.0534$

- $B_{3}=58.16$.

Calculations results for the effective thermal conductivity for the analyzed bundles are presented in Fig. 7. The obtained $k_{\text {ef }}$ values for the $60 \mathrm{~mm}$ sections are within the $3.9 \div 8.8 \mathrm{~W} /\left(\mathrm{m}^{2} \cdot \mathrm{K}\right)$ range, whereas for the $80 \mathrm{~mm}$ sections the $k_{\mathrm{ef}}$ values are within the $3.5 \div 11.3$ $\mathrm{W} /\left(\mathrm{m}^{2} \cdot \mathrm{K}\right)$ range. This coefficient increases as a function of temperature, and the dynamic of it increases with the increase in the emissivity. At the same time, higher values were obtained when the radiation resistance $R_{\text {rd-ap }}$ was taken into account in the calculations. 

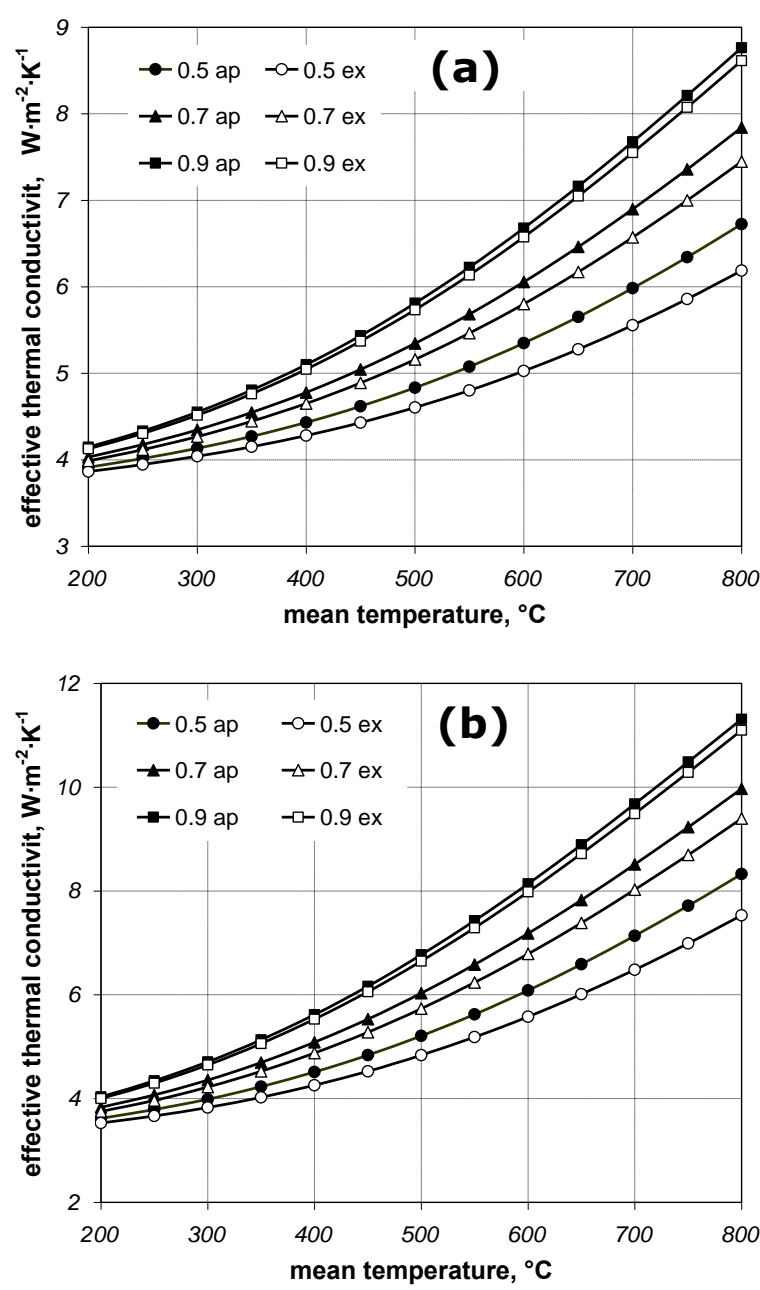

Fig. 7. The coefficient $k_{\text {ef }}$ calculated with both the exact and the approximate methods of the radiation resistance, results for: a) $60 \mathrm{~mm}$ section bundle, b) $80 \mathrm{~mm}$ section bundle.

In order to demonstrate the influence of the radiation resistance value on the effective thermal conductivity, the percentage difference between the $k_{\text {ef }}$ values obtained for both scenarios was calculated. This parameter is defined as follows:

$$
\Delta k_{e f}=\frac{k_{e f-a p}-k_{e f-e x}}{k_{e f-e x}} \cdot 100 \%
$$

where $k_{\text {ef-ap }}$ denote the $k_{\text {ef }}$ value calculated taking into account the resistance $R_{\text {rd-ap }}$, and $k_{\text {ef-ex }}$ denote the $k_{\text {ef }}$ value calculated taking into account the resistance $R_{\text {rd-ex }}$. The results of the calculations for the $\Delta k_{\mathrm{ef}}$ for each bundle are presented in Fig. 8 .

The value of the $\Delta k_{\text {ef }}$ for the $60 \mathrm{~mm}$ section bundle in $200^{\circ} \mathrm{C}$ temperature was not greater than $1.5 \%$. However, when the temperature increased to $800^{\circ} \mathrm{C}$ the obtained values were between $1.7 \%$ and $8.7 \%$. The highest values were observed for the emissivity of 0.5 . For the emissivity of 0.7 the maximum value was $5.3 \%$. A similar trend was observed for the $80 \mathrm{~mm}$ sections. For $200^{\circ}$ the maximum value obtained was $2.6 \%$, whilst for $800^{\circ} \mathrm{C}$ and the successive emissivities obtained values were $10.6 \%, 6.1 \%$ and $1.9 \%$ respectively.
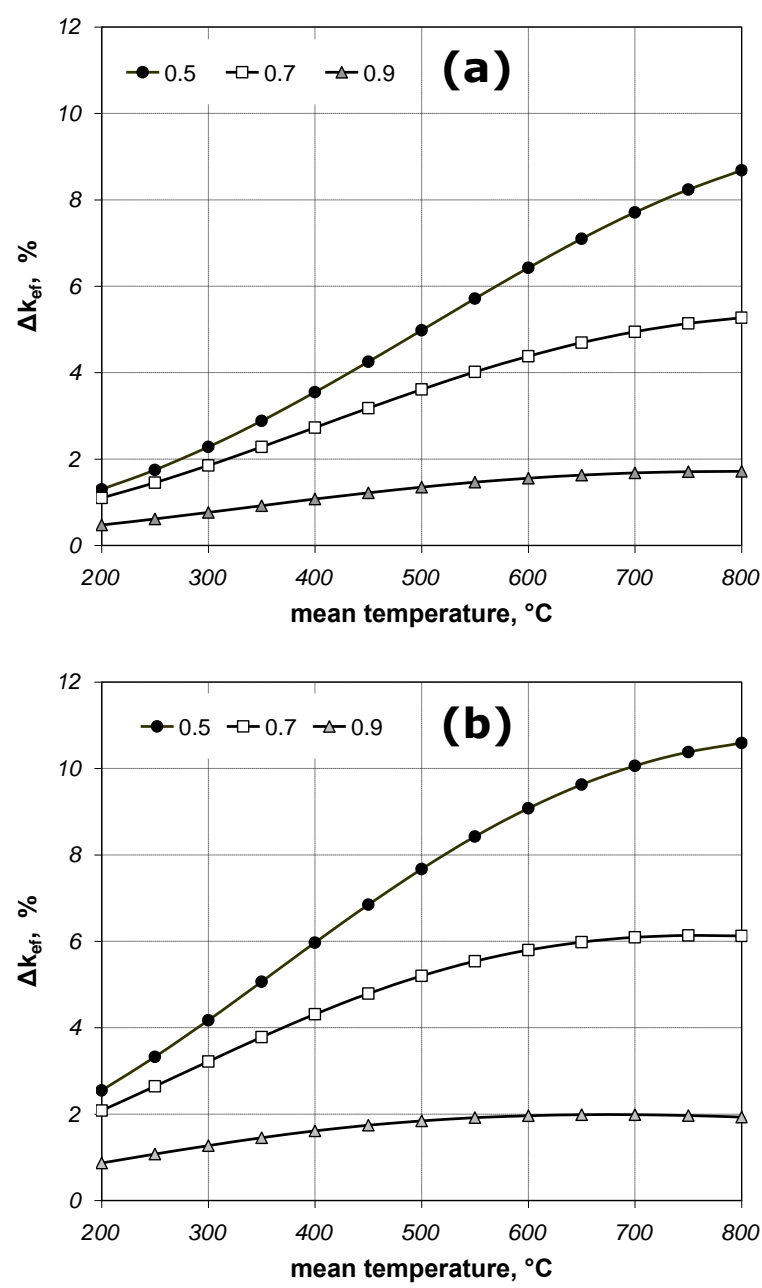

Fig. 8. Values of the $\Delta k_{\text {ef }}$ parameter for: a) $60 \mathrm{~mm}$ section bundle, b) $80 \mathrm{~mm}$ section bundle.

The values of the $\Delta k_{\text {ef }}$ parameter averaged for the whole temperature range obtained for all the analyzed scenarios are summarized in Table 1.

Table 1. The averaged values of $\Delta k_{\text {ef }}$ for each bundles.

\begin{tabular}{|c|c|c|}
\hline Emissivity & Sections $60 \mathrm{~mm}$ & Sections $80 \mathrm{~mm}$ \\
\hline 0.5 & $5.0 \%$ & $7.2 \%$ \\
\hline 0.7 & $3.4 \%$ & $4.7 \%$ \\
\hline 0.9 & $1.2 \%$ & $1.6 \%$ \\
\hline
\end{tabular}

As it can be seen, the use of the approximate method for calculations the radiation resistance in the model of the effective thermal conductivity, in relation to the entire temperature range, exceeds the maximum value of the $k_{\text {ef }}$ coefficient by approximately $7 \%$. However, this applies to the emissivity of 0.5 , while the surface of steel profiles subjected to heat treatment is usually characterized by the emissivity above 0.7 . Therefore, the overestimation of the value of $k_{\text {ef }}$ using the approximate method will be less than $5 \%$. It can be stated that for industrial needs, the accuracy of calculations at this level is sufficient. 
However, in a situation where more accurate calculations of the $k_{\mathrm{ef}}$ coefficient are required, the radiation resistance can be calculated using the approximate method. In order to apply this approach, the use of an appropriate correction term is required, which takes into account the linear impact of the emissivity:

$$
R_{r d-c r}=(1.39-0.39 \varepsilon) \frac{X_{r d}}{4 \sigma T_{m}^{3}}
$$

Due to the use of equation (17), the mathematical model of the effective thermal conductivity avoids solving the system of equations (6a), however, the results of these calculations will not be overstated as observed with equation (7).

\section{Conclusions}

The problem presented in this article is related to the optimization of heat treatment of the square steel profiles heated in the form of bundles. Due to the porous structure of this charge, its basic thermal property is effective thermal conductivity. This parameter quantifies the ability of the bundle to transmit heat as a result of the complex processes of conduction in steel and air, contact conduction, thermal radiation and free convection. The calculations of the $k_{\text {ef }}$ coefficient were performed for two scenarios, which differ in the way of their modeling (exact and approximate) for radiation resistance. It has been shown that with the approximate method of determining resistance $R_{\mathrm{rd}}$, the results of the $k_{\mathrm{ef}}$ calculations are overstated by an average of about $5 \%$. In order to eliminate this discrepancy an updated version of the approximate equation was proposed, in which a corrective term was added. This term takes into account the linear impact of emissivity on the value of radiation resistance $R_{\text {rd }}$.

\section{References}

1. T.R. Rao, G.J. Barth, J.R. Miller, Iron and Steel Eng. 60 (1983)

2. Y. Jaluria, Int. J. Num. Met. Eng. 25 (1988)

3. S. Sahay, R. Mehta, K. Krishnan, Mat. Man. Proc. 22 (2007)

4. M. Kaviany, Principles of heat transfer in porous media ( $2^{\text {nd }}$ ed. Springer-Verlag, New York, 1995)

5. W van Antwerpen, C.G. du Toit, P.G. Rousseau, Nuc. Eng. Des. 240 (2010)

6. D. Kunii, J.M. Smith, AIChE J., 6 (1960)

7. P. Zehner, E.U. Schlunder, Chem. Ingr. Tech. 42 (1970)

8. F.M. Modest, Radiative heat transfer ( $3^{\text {rd }}$ ed. Academic Press, Amsterdam, 2013)

9. R. Wyczolkowski, D. Urbaniak, J. Ther. Heat Trans. 30 (2016)

10. Y.A. Cengel, Heat and mass transfer, a practical approach ( $3^{\text {rd }}$ ed. McGraw-Hill, New York, 2007)
11. R. Wyczolkowski, Proc. Eng. 157 (2016)

12. R. Wyczolkowski, H. Radomiak, T. Wylecial, Proceedings of $10^{\text {th }}$ International Conference on Computational Heat, Mass and Momentum Transfer (ICCHM2T 2017, May 28-June 1, Seoul, Korea, Paper Number 160149)

13. R. Wyczolkowski, A. Benduch, Civ. Env. Eng. Rep. 14 (2014)

14. T. Senkara, Thermal calculations of furnaces in metallurgy (Wydawnictwo Śląsk, Katowice, 1981 in Polish)

15. K. Raźnjević, Thermal tables with diagrams (WNT, Warsaw, 1966 - in Polish) 